\title{
Design of Intelligent Home Anti-theft Alarm System
}

\author{
Zhao $\mathrm{He}^{1, \text { a }}$, Yu Jun $\mathrm{Qi}^{2, \mathrm{~b}}$ \\ ${ }^{1}$ Electronic and Information Engineering School of Lanzhou Jiaotong University, No. 88, Anning \\ Road West Side, Anning District, Lanzhou, Gansu,China 730070 \\ ${ }^{2}$ Electronic and Information Engineering School of Lanzhou Jiaotong University, No. 88, Anning \\ Road West Side, Anning District, Lanzhou, Gansu,China 730070 \\ azhk98@mail.Izjtu.cn, byujunqi@mail.Izjtu.cn
}

Keywords: microcontroller, sensors, central alarm processing unit, anti-theft alarm, network communication

Abstract. In view of the situation of home security problems and the defect of security products in
the domestic market, a new intelligent home anti-theft alarm system is designed by using of ATMEL
SAM R21 microcontroller, multiple and various detection sensors and central alarm processing unit.
The network communication mode adopts Atmel's own Lightweight Mesh protocol between sensors
and the central alarm processing unit, which has the characteristics of high security, stable and
reliable. The central alarm processing unit can give sound, light, SMS and phone alarm, when it
receives the intrusion information from front sensors. The advantages of the system are low cost,
perfect function, simple to install and convenient to extend. The system can be widely used in family.

\section{Introduction}

In recent years, a large number of modern residential district have been completed in our country, at the same time, the population mobility increases, and the public security situation is becoming more and more complicated, therefore, home security issues is particularly important. Almost $70 \%$ of the family has installed anti-thief doors and burglar mesh in the domestic large and medium-sized cities, while very few families install anti-thief doors and burglar mesh in developed countries such as America, Japan, and etc. Burglar mesh is not only unbeautiful, but also maybe lead to potential safety hazard in case of fire or other unexpected events. A perfect intelligent home anti-theft alarm system can ensure safety of each family easily [1].

Many families have installed intelligent home anti-theft alarm system in high quality residential area or villas, the cost of the system is very high, ordinary wage earners cannot afford the price. They need these kinds of intelligent security products which are cheap, easy to use and convenient to expand. At present, the price of home security products on the market is between $100 \mathrm{RMB}$ and 1000 RMB, the common problems are: (1) Wireless communication mode uses $315 \mathrm{MHZ}$ or $433 \mathrm{MHZ}$, the security is poor, the capacity of coding is small, and the system is vulnerable to electromagnetic interference. (2) The power consumption is large, and it needs to replace the battery frequently. (3) When the alarm host or gateway fails, the entire system will break down. (4) Its function is limited, and the function of extension interface does not be provided in this system.

This paper puts forward a design scheme of a new intelligent home anti-theft alarm system to solve the above problems.

\section{The overall design scheme of the system}

The system consists of four parts that are central host, wireless gateway, front-end detectors (the human body sensor and door magnetic sensor) and wireless switch. The overall block diagram of the system is shown as in Fig.1. 


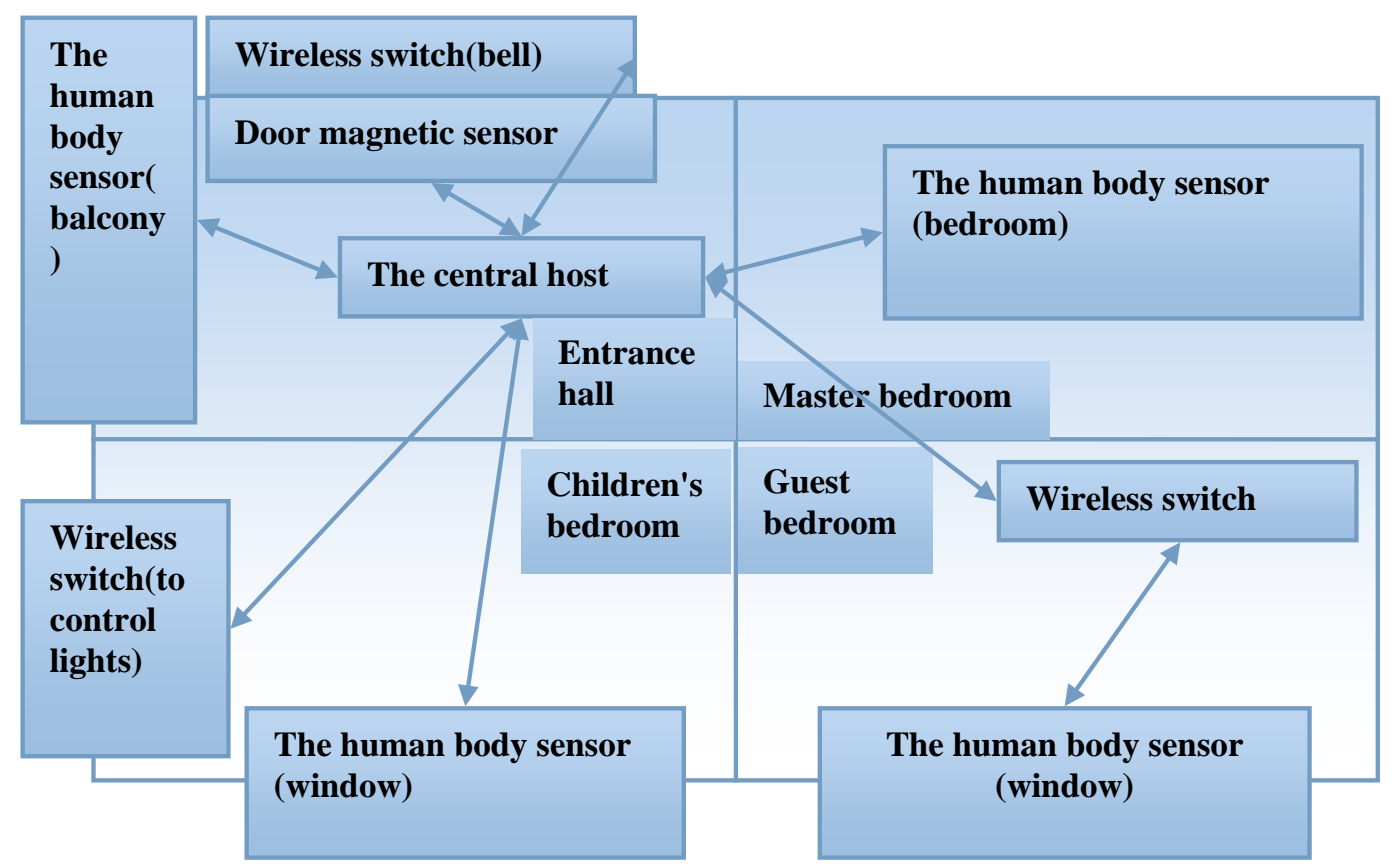

Fig. 1 The overall block diagram of the system

The central host applies ATMEL SAM R21 Xplained Pro. Wireless communication mode adopts ATMEL Lightweight Mesh protocol based on IEEE 802.15.4. In this way, the communication security can be improved and the power consumption can also be reduced, on the other hand, the ad-hoc network between the components can be realized easily, function module from different department will still work normally, even when the central host fails.

Two measures are used to realize its further function extension conveniently. Firstly, the characteristic of the ad-hoc network makes it possible to join a new function module later. Secondly, wireless switch components are included in this system, they provide high flexibility for function extension, and the advantages of ATMEL products can be made full use of.

In Fig.1, double arrow lines show wireless communication lines, other components are introduced as follows:

(1) Door magnetic sensor: it is generally installed on the door and door frame, the central host will be triggered and display alarm location when thieves break into the house illegally [2].

(2) The human body sensor: it is mainly installed near the Windows and balconies in order to detect illegal intruder with method of infrared, it can also be installed in the inner regions of the sitting room or bedroom in order to start or trigger LED night light in these place.

(3) Wireless switch: it is used to control function devices to work and close, the purpose of it is the same with common switch, and there are two applications in the system:

Firstly, switch signals from buttons are sent out by wireless communication, other matching function module will control the module to work or stop after receiving the signal. The wireless switch can be used as a doorbell, when a visitor presses the bell, press information will be sent to the central host to make the doorbell ring.

Secondly, it can also be used to control relay by wireless communication, then other function device will be further drove to work or stop. On this occasion, power lines of controlled device such as Neutral wire need to be connected into wireless switch, live wire should be connected into the controlled device for security purposes.

(4) Wireless gateway: it is only used for relay nodes of wireless communication in order to expand the range of front-end detectors, small family don't need this component.

(5) The central host: it is used to collect and process information from front-end detectors, and to determine whether to give alarm signals. 


\section{System function}

(1) Wireless switch can be set to the mode of doorbell. When a visitor presses the wireless switch, the central host will play the music of doorbell to tell the homeowner a guest coming to visit after receiving the information.

(2) The users can set the system to protection mode by SMS or the work mode button on the central host when they go out. If a thief enters, the human body sensor or door magnetic sensor will immediately detect the information and trigger the central host to give alarm. There are four kinds of alarm modes designed in the system including SMS alarm, phone alarm, sound and light alarm. Alarm principle is made by this way, the remote alarm by SMS or phone is firstly been sent out, then local alarm by sound and light will be given [3].

(3) To avoid false alarm, the users need to remove protection by SMS or the work mode button on the central host before entering into the house, the function of removing protection can also be completed by wireless switch (door bell), and a keyboard will be configured on wireless switch to input password of removing protection.

(4) The mode of protection will automatically start in the period of time setting by the users, such as night time.

(5) Each node of the system can be configured by SMS, and all configuration information can be queried by SMS.

\section{Hardware circuit design of the system}

SAM R21 Xplained Pro is chosen as main microcontroller, it includes 32-bit ARM Cortex-M0+ microprocessor and the best 2.4 G RF transceiver for IEEE 802.15.4. It provides the highest RF performance between single chip equipment of industry, and at the same time, its current consumption is only $50 \%$ of the existing products. The highest frequency can reach $48 \mathrm{MHZ}$ [4]. The lightweight mesh protocol developed by Atmel Corporation is simple to implement and open to use, the communication between front-end detectors and alarm host adopts the protocol in system design. GPRS module sends alarm information to the user's mobile, and receives control information from the user's mobile. MC35 module of Siemens Corporation is used in the system that supporting many kinds of communication mode, such as data, voice, SMS and FAX, AT command can be applied to control the MC35 module [5]. Serial line is transformed by MAX3245 chip in order to directly connect to RS232 serial port.

(1) Front-end detectors

Front-end detectors contain only the human body sensor and magnetic sensor [6], front-end detectors connection diagram is shown as in Fig.2.

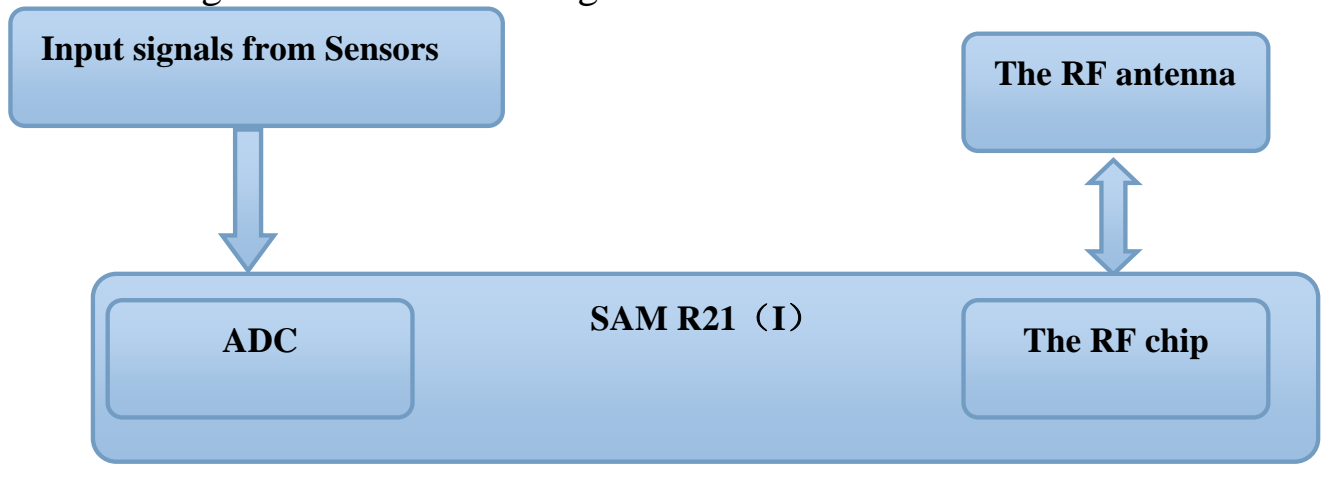

Fig.2 Front-end detectors connection diagram

The difference from door magnetic sensor in the system is that the human body sensor has two kinds of deployment pattern. The pattern 1 is used for anti-theft, and the pattern 2 is used for automatic night light. Therefore, the human body sensor needs to include light detection sensor, dial switch is used to change the two modes. 
(2) Wireless switch

The purpose of wireless switch is mainly for further expansion, wireless switch is used as trigger device, and the function of it is equal to key switch. Wireless switch connection diagram is shown as in Fig.3.

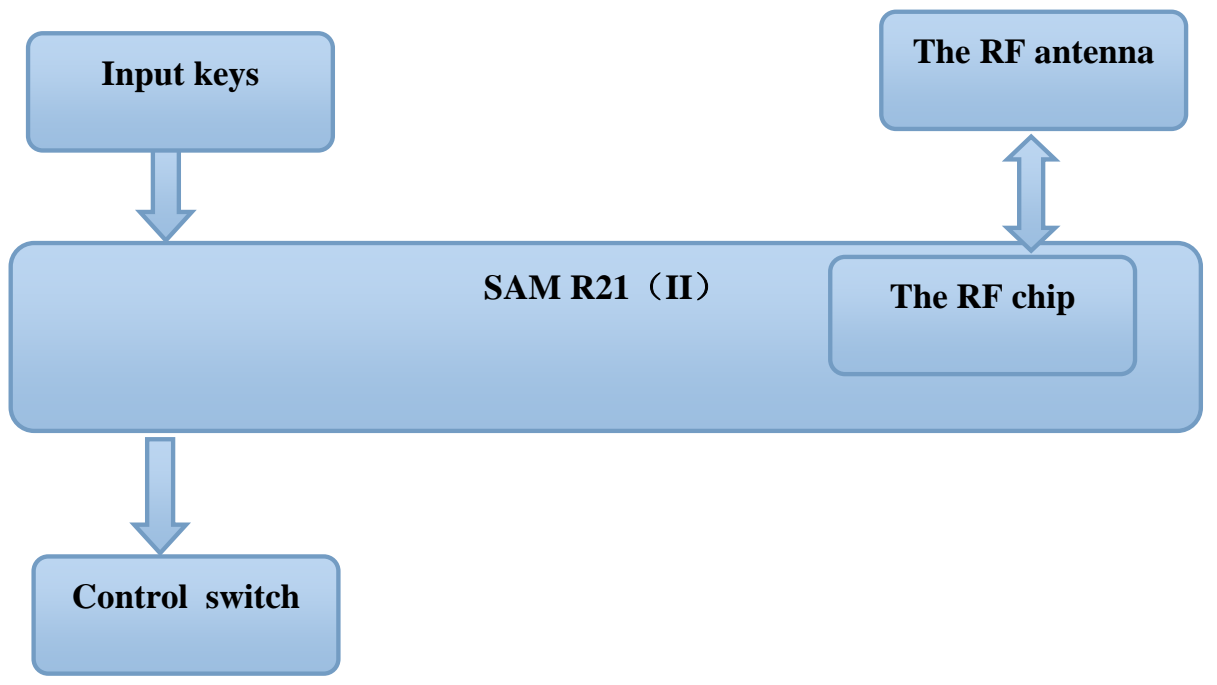

Fig.3 Wireless switch connection diagram

(3) Wireless gateway

As wireless communication relay nodes, wireless gateway is used to extend the layout range of front detectors, and it is suitable for large room. Wireless gateway connection diagram is shown as in Fig.4.

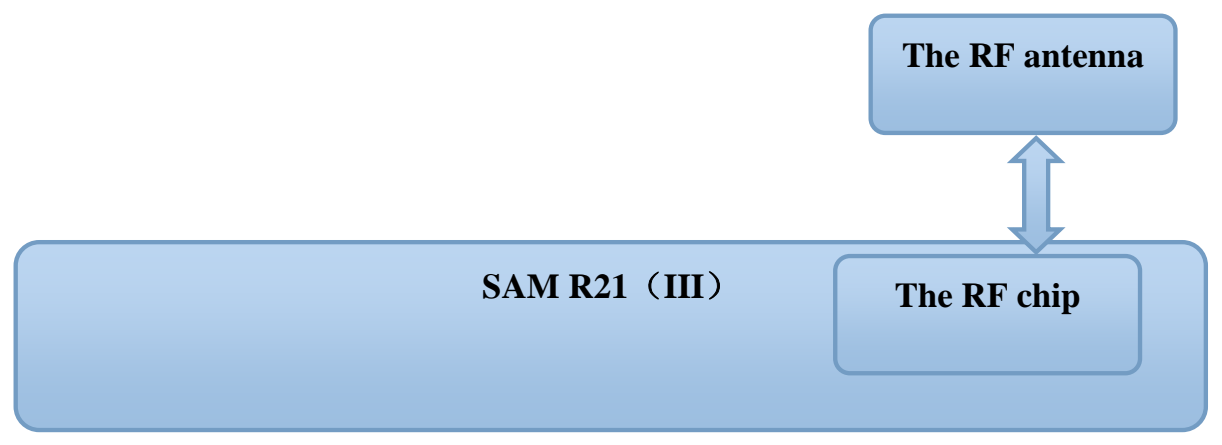

Fig.4 Wireless gateway connection diagram

(4) The central host

The central host is the main processing unit in the system. It is responsible for handling information from front-end detectors and judges whether to give alarm. In order to reduce the cost of the system, all configuration information can be set and queried by GRPS SMS, it doesn't need to design special input and output devices in the system.

Simple keys designed in the system are used to set working mode. Reset button is used to restore default Settings, and led lights are used to display according working mode. The central host communicates with wireless gateway or front-end detectors by Lightweight Mesh protocol, and it stores Data that need to be recorded into SD Card. The central host circuit connection diagram is shown as in Fig.5. 


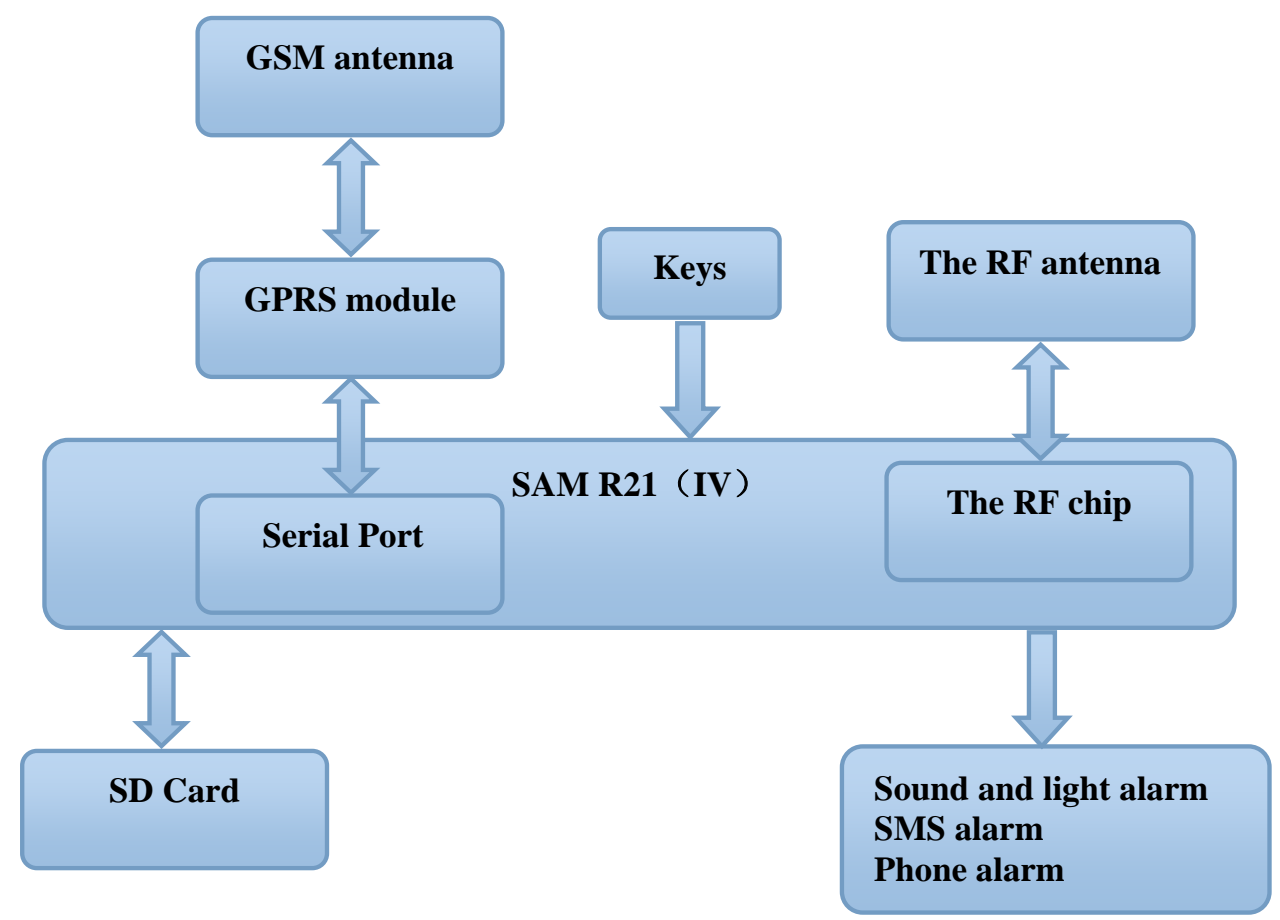

Fig.5 The central host circuit connection diagram

\section{Software design of the system}

A reliable microcomputer application system is built by use of various intrusion detection sensors and MCU and etc. the human-computer interaction function can be realized by SMS between a mobile phone and the central host. The function of front-end detectors is simple, and the corresponding software logic is relatively simple, the paper mainly introduces software design for the central host.

When powered, the central host carries out a self-check. Self-check includes detection for its hardware condition and its communication with each component configured. After the detection passes, the central host will enter into work cycle, three functions can be performed: (1) Input signals from sensors are collected continuously and transferred into microcontroller to calculate, the alarm condition designed in software design is that a sensor is triggered after deployment, if the condition meets, alarm will be given. (2) Information from SMS can be received and analyzed, the function of configuring and querying SMS can be completed. (3) Wireless switch is used as the function of doorbell.

The main program flow chart is shown as in Fig.6.

\section{Summary}

In order to solve problems existed in products of anti-theft alarm system, an intelligent home anti-theft alarm system based on ATMEL SAMR21 Xplained Pro is designed in this paper, the system makes full use of the advantages of ATMLE low power consumption and RF technology, it has good practicability , flexible scalability, and strong anti-interference ability. The use of various front-end detectors can effectively prevent false alarm. 


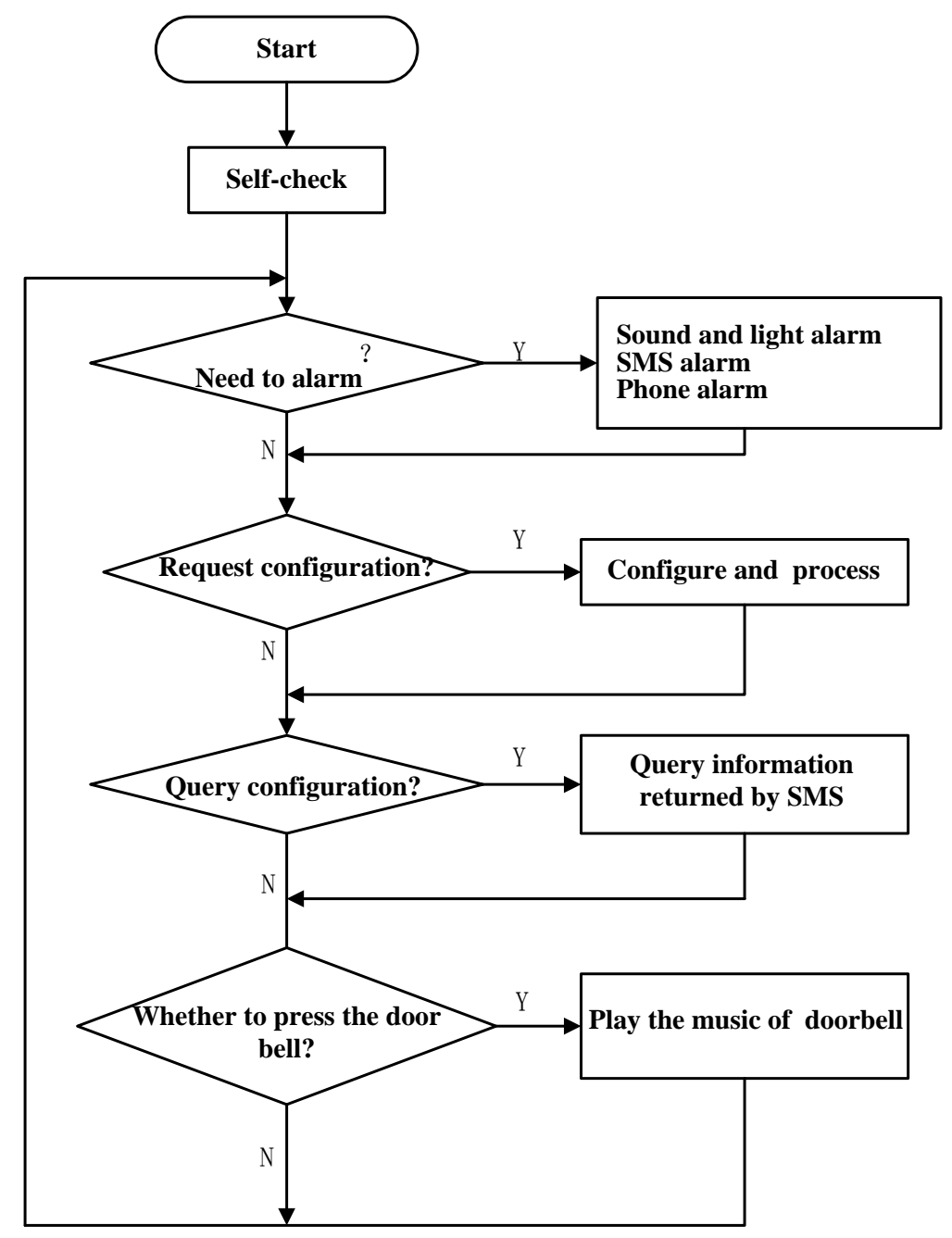

Fig.6 The main program flow chart

\section{References}

[1] Liu Shengwu, FanHong and Cai Weifeng: Smart Home Burglar Alarm Subsystem Based on ARM+Zigbee. Computer Systems \& Applications Vol.24 No.2 (2015), p.257- p.260

[2] Wang Songde, Zhao Yan, Yao Liping, and Zhang Shuanji: Digital Acoustic Burglar Alarm System Using Infrared Radio Remote Control. Spectroscopy and Spectral Analysis Vol.29 No.3 (2009), p.858- p.861

[3] Zhang Sen: Optical Fiber Intelligent Burglar Alarm System. Optical Communication Technology No.11 (2013), p.34- p.36

[4] Shen Jianhua, and Hao Liping: ARM Cortex-M0+ Principle and Application of Microcontroller Based on The Series of ATMEL SAM D20 (Beihang University Press,China 2014).

[5] Zhao Ting: Design of Smart Vehicle Anti-theft System Based on Vehicle Networking. Application of Electronic Technique Vol.41 No.3 (2015), p.61- p.64

[6] Zhao yu-an: Wireless Door Magnetic Sensor. Electronics DIY No.11 (2004), p.40- p.41 